\title{
Lymphogranuloma Venereum Presenting as a Rectovaginal Fistula
}

\author{
C.M. Lynch,* T.L. Felder, R.A. Schwandt, and R.G. Shashy \\ Department of Obstetrics and Gynecology, University of South Florida College of Medicine, \\ Tampa, FL
}

\begin{abstract}
Lymphogranuloma venereum (LGV) is a rare form of the sexually transmitted disease caused by Chlamydia trachomatis. In the United States, there are fewer than 350 cases per year. In a review of the world's literature, there has not been a case reported in the last thirty years of a case of LGV presenting as a rectovaginal fistula. We present a case of an otherwise healthy American woman who presented with a rectovaginal fistula. Although uncommon, LGV does occur in developed countries and may have devastating tissue destruction if not recognized and treated before the tertiary stage. Infect. Dis. Obstet. Gynecol. 7:199-201, 1999. @ 1999 Wiley-Liss, Inc.
\end{abstract}

sexually transmitted disease, fistula, lymphogranuloma venereum

\section{INTRODUCTION}

Lymphogranuloma venereum (LGV) has a reported incidence of approximately 350 cases per year in the United States and rarely presents in its tertiary or anorectogenital stage. ${ }^{1}$ We present a case of a woman with a rectovaginal fistula due to LGV.

\section{CASE REPORT}

A 43-year old black female, gravida 2 para 2, presented with a 2-month history of passing stool through her vagina. She also noted increasing vulvar swelling and discomfort over the last several months. She stated that approximately three years ago she had a swollen right lymph node in the groin region, which was surgically drained and then resolved. An edematous process began again approximately 6 months ago, which now included her entire vulva. She had no significant past medical history. Her past surgical history was significant only for a nonmalignant right breast biopsy for a nontender mass. She had a history of normal monthly periods at regular intervals and denied a history of sexually transmitted diseases or abnormal pap tests. She was married and stated that she had been monogamous for six years. There was no significant family history. The patient smoked one pack per day for 25 years and drank one liter of alcohol per week. She denied illicit drug use. Physical examination revealed an afebrile $5^{\prime} 4^{\prime \prime}, 132-1 \mathrm{lb}$. woman with blood pressure of 106/48. General physical exam and mental status exam were normal. No skin abnormalities were noted with the exception of the pelvic findings. The pelvic exam revealed enlarged, irregular, edematous labia, bilaterally, with complete distortion of the right labia majora due to edema and fecal soiling (Fig. 1). Tissue bridges and draining sinuses were visually noted as well as a rectovaginal fistula and several blind tracts (Fig. 2). An internal examination was unable to be performed secondary to pain. Consultations with specialists from gynecologic oncology, colorectal surgery, and infectious disease were obtained. Af-

*Correspondence to: Catherine M. Lynch, MD, University Of South Florida College Of Medicine, Department Of Obstetrics and Gynecology, 4 Columbia Drive, Suite 500, Tampa, FL 33606. 


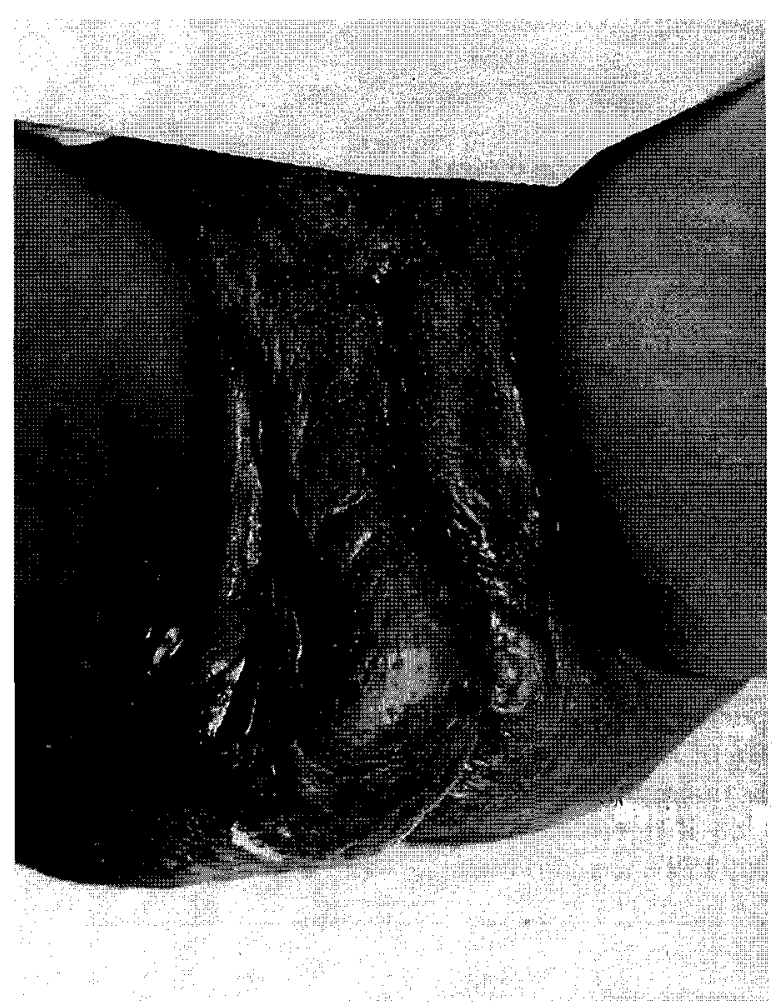

Fig. I. Vulva and perineum. Right labia with distortion from lymphedema.

ter consultation the differential diagnosis included hidradenitis suppurativa, LGV, and vulvar or rectal cancer.

Biopsies and a wound culture as well as titers for Chlamydia, Nocardia, and acid-fast bacilli were taken. The patient was empirically started on doxycycline $100 \mathrm{mg}$ p.o. twice a day due to the likelihood of infectious etiology. Serology was positive only for Chlamydia by complement fixation (titer at $1: 8$ with reference range $<1: 8$ ). Biopsy showed benign squamous epithelium with submucosal chronic inflammation.

A colostomy was performed one week after initial evaluation along with an examination under anesthesia. There were visible draining sinuses in the perirectal area. Proctoscopy examination revealed involvement of anal margin but did not extend beyond the anal verge. A large LGV lesion was visible. The area was erythematous and edematous with pocking, and there were several sinus tracts in the involved area. The rest of the anal canal was essentially normal.

The patient did well postoperatively, with the exception of a complaint of yellow-green vaginal discharge six weeks postoperatively. Wet prep evaluation revealed Trichomonas that was treated with metronidazole.

Seventeen weeks after the first surgery, the patient underwent a partial vulvectomy, repair of rectovaginal fistula, and debridement of the remainder of the affected perineum. One week later, a split-thickness skin grafting to the debrided area was performed.

Three months later, the patient underwent closure of the colostomy after the skin graft had healed. At this time, there was no evidence of fistula in the rectum or vagina.

\section{DISCUSSION}

LGV is rare in North America with fewer than 350 cases per year in the United States. ${ }^{1}$ The causative organism for LGV is Chlamydia trachomatis serotypes L1, L2, and L3. The incubation can last from a few days to 21 days. The primary lesion of this disease is a painless ulcer, which often goes unnoticed and resolves spontaneously. In the following weeks, the secondary stage presents as a painful lymphadenopathy in the inguinal lymph nodes develops. In the absence of treatment, the overlying skin becomes brawny and wrinkled with a characteristic violaceous hue, and the abscesses within the nodes coalesce and drain from one or more sinus tracts. The tertiary stage involves perirectal abscesses and rectal strictures and fistulas. This stage is usually seen in women and homosexual men. The patient usually has a mild leukocytosis with an elevation in monocytes and eosinophils. ${ }^{2}$ LGV can be diagnosed though serologic detection of antibodies to $C$. trachomatis, complement fixation, DNA probe, lymph node culture, or physical examination. ${ }^{3}$ Due to the lack of specificity and sensitivity of the serologic test for Chlamydia species, a thorough physical exam often confirms the diagnosis.

In this patient, the differential diagnosis included hidradenitis suppurativa, and rectal or vulvar carcinoma. The vulvar biopsy in our patient, however, revealed edematous tissue that was not purulent or sebaceous in character. Since she also had a positive Chlamydia-specific antibody test, a diagnosis of the tertiary stage of LGV was made.

Treatment of LGV includes a three-week course of a tetracycline or erythromycin (or azithromycin), $500 \mathrm{mg}$ four times a day. ${ }^{4}$ Sulfadiazine may be useful and is administered by giving a loading 


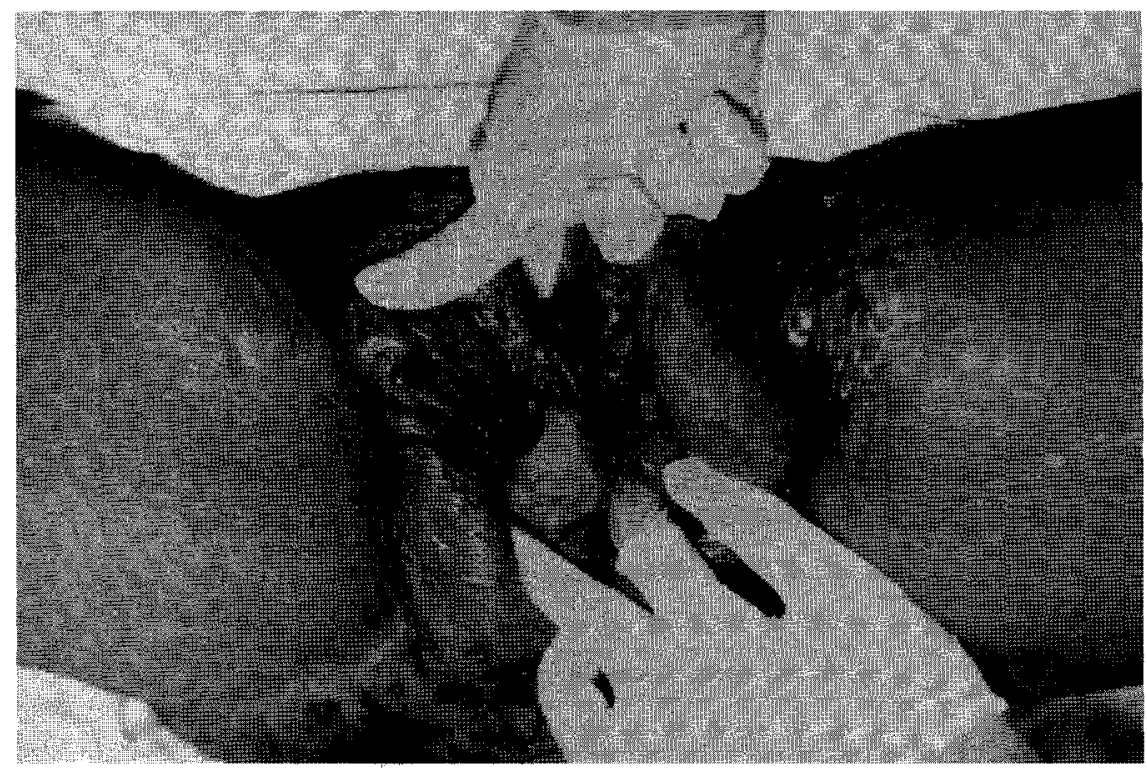

Fig. 2. Operator's fingertip visible through rectovaginal fistula. Multiple sinus tracts visible.

dose of $2 \mathrm{~g}$ followed by $4 \mathrm{~g}$ a day for $10-28$ days. Erythromycin or azithromycin would be indicated in pregnancy. In this case, a six-week course was given because of tissue induration and suspected poor perfusion with gross fecal contamination. The LGV lesion improved with doxycycline, but the extensive involvement of the perineum mandated surgical as well as medical therapy. Follow-up care was needed to repair the damage caused by the lymphedema. Surgical debridement is recommended in this type of presentation. ${ }^{5}$

Although LGV is rare is the United States in 1988, Epling and Reich reported an outbreak of LGV in Central Florida linked with prostitution. ${ }^{6}$ In fact, two months after this presentation, a second woman came to this clinic with a case of secondary LGV. Although, LGV is a rare disease in this country, it can be carried due to global travel, migration, and immigration. Because this disease starts as a painless ulcer and resolves rather quickly, it is often ignored in the primary stage. Because of this seemingly harmless first stage, this disease can be transmitted unknowingly to other people. ${ }^{7}$ Although it is rare in developed countries, all clinicians should be mindful of this entity in order to prevent the spread and the destructive tertiary stage of this disease.

\section{REFERENCES}

1. Sweet RL. Infectious Diseases of the Female Genital Tract. $3^{\text {rd }}$ Ed. Baltimore, MD: Williams \& Wilkins, 1995.

2. Abrams AJ. Lymphogranuloma venereum. JAMA 1968; 215:199.

3. Goens JL, Schwartz RA, DeWolf K. Mucocutaneous manifestation of chancroid, lymphogranuloma venereum, and granuloma inguinale. Am Fam Phys 1994;49: 415-425.

4. Faro S. Lymphogranuloma venereum, chancroid and granuloma inguinale. Obstet Gynecol Clin North Am 1989;16:517-529.

5. Hirschberg SM, Horton CE. Radical perineal resection for far advanced lymphogranuloma venereum. Plast Reconstr Surg 1973;51:217-219.

6. Epling S, Reich GA. Report on an Outbreak of lymphogranuloma venereum in central Florida. J Fla Med Assoc 1988;75:29-30.

7. Fitzpatrick TB, Eisen AZ, Wolff K, Freedberg IM, Austen KF. Dermatology in General Medicine. 4th ed. McGraw-Hill, 1993. p. 761-765, 2753-2756. 


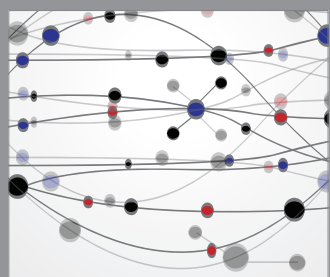

The Scientific World Journal
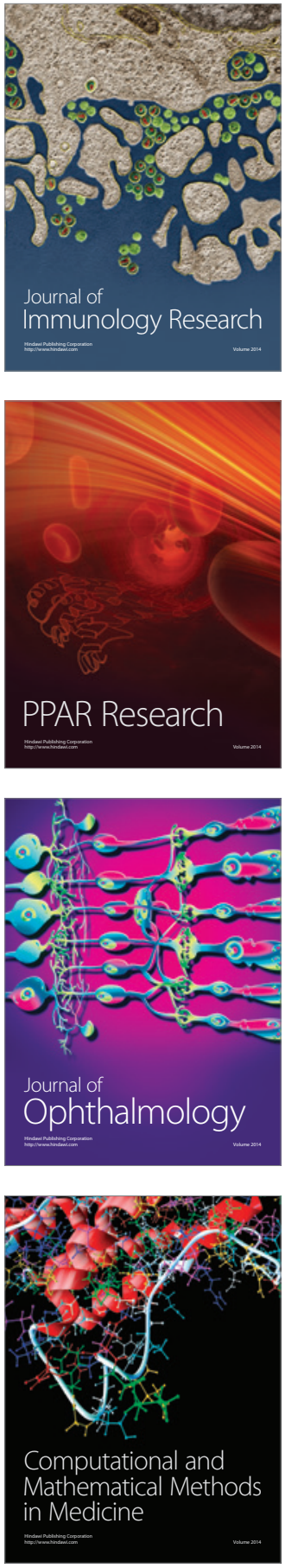

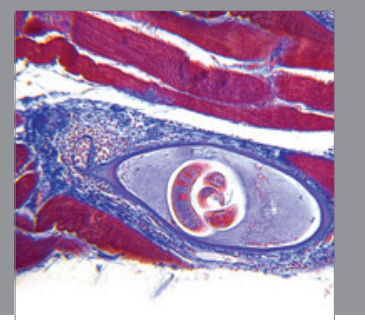

Gastroenterology

Research and Practice
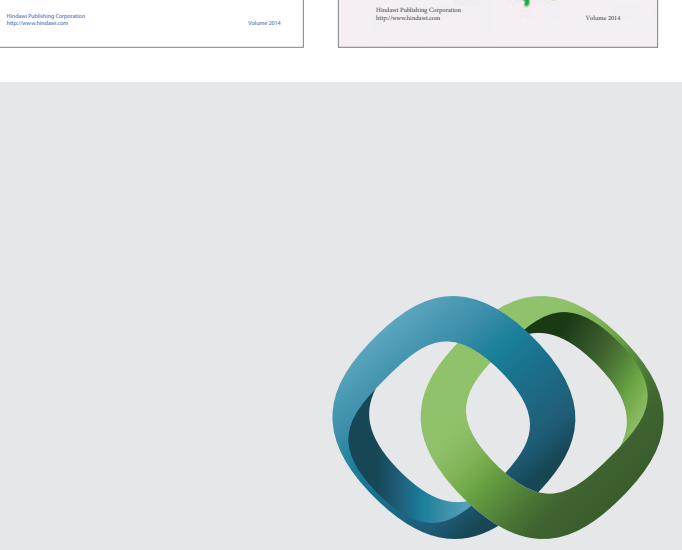

\section{Hindawi}

Submit your manuscripts at

http://www.hindawi.com
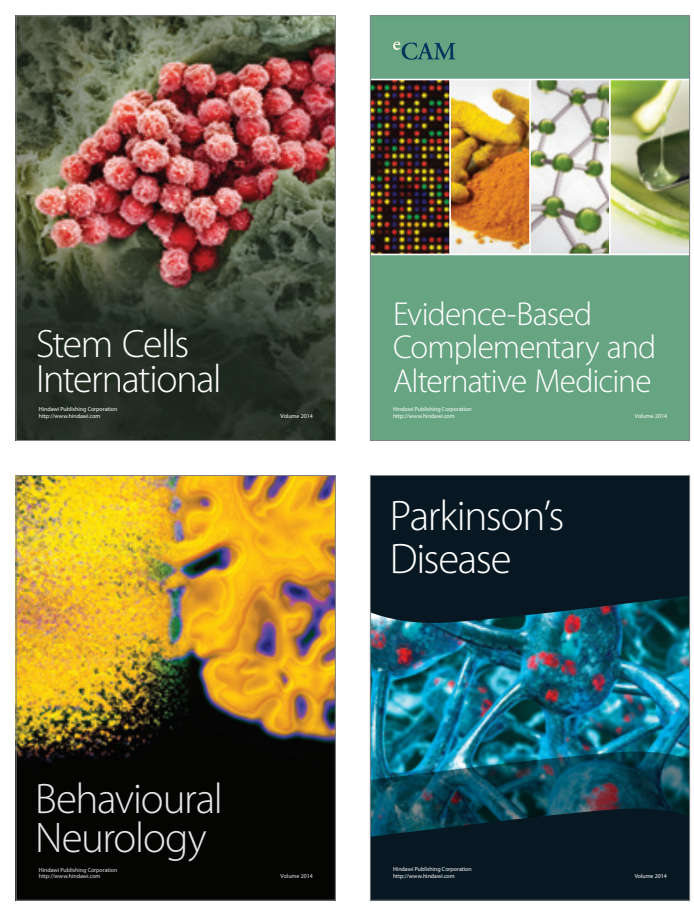

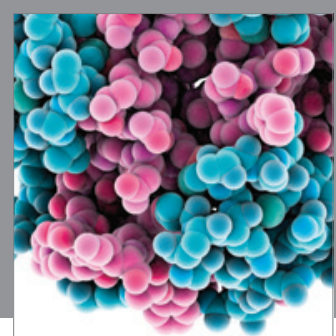

Journal of
Diabetes Research

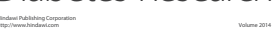

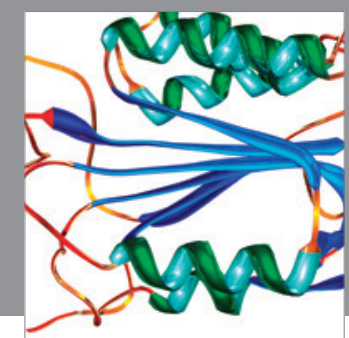

Disease Markers
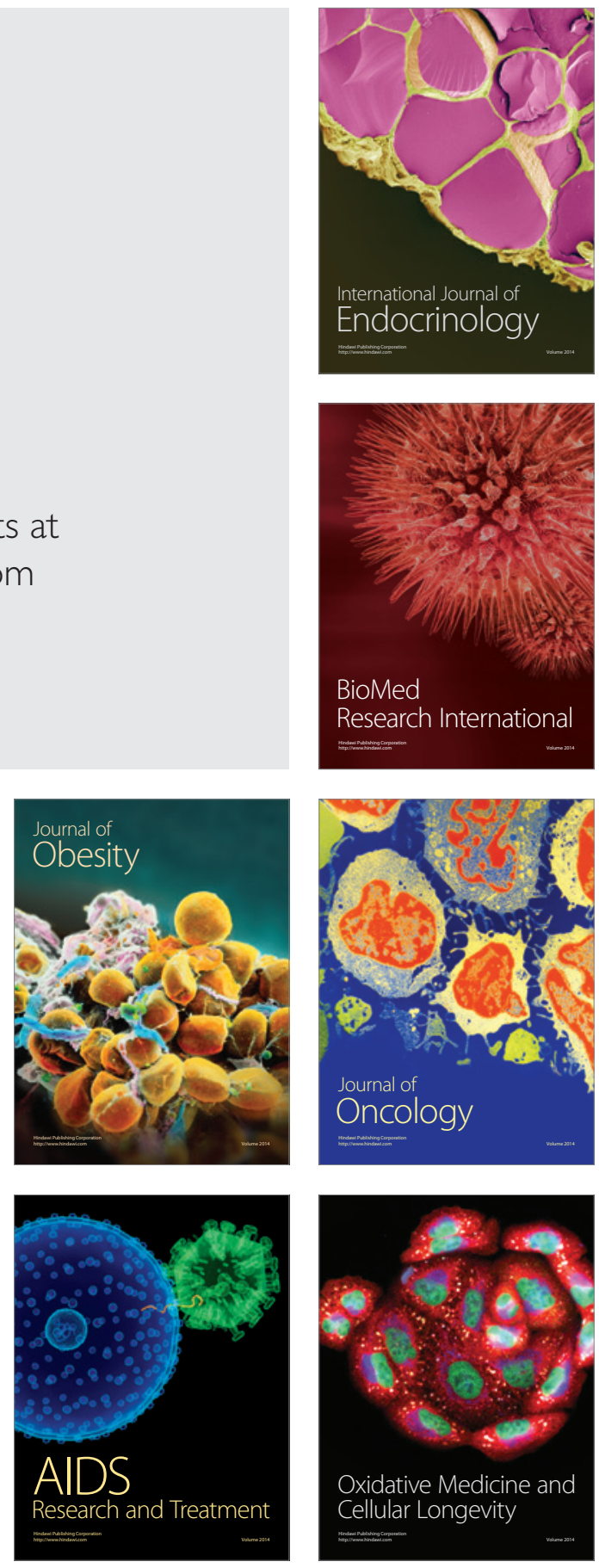